\title{
Exploring the Metabolomic Responses of Bacillus licheniformis to Temperature Stress by Gas Chromatography/Mass Spectrometry ${ }^{[}$
}

\author{
Zixing Dong', Xiaoling Chen ${ }^{2}$, Ke Cai ${ }^{2}$, Zhixin Chen ${ }^{2}$, Hongbin Wang ${ }^{2}$, Peng Jin ${ }^{1}$, Xiaoguang Liu ${ }^{1}$, \\ Kugenthiren Permaul ${ }^{3}$, Suren Singh ${ }^{3}$, and Zhengxiang Wang ${ }^{1,2 *}$ \\ ${ }^{1}$ College of Chemical Engineering and Materials Science, Tianjin University of Science E Technology, Tianjin 300457, P.R. China \\ ${ }^{2}$ Key Laboratory of Industrial Fermentation Microbiology, Ministry of Education, College of Biotechnology, Tianjin University of Science E \\ Technology, Tianjin 300457, P.R. China \\ ${ }^{3}$ Department of Biotechnology E Food Technology, Faculty of Applied Sciences, Durban University of Technology, Durban 4001, South Africa
}

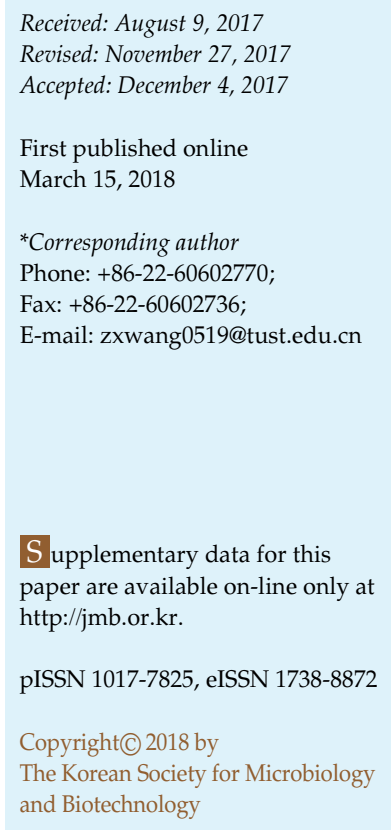

S upplementary data for this paper are available on-line only at http://jmb.or.kr.

pISSN 1017-7825, eISSN 1738-8872

Copyright(C) 2018 by

The Korean Society for Microbiology and Biotechnology

\begin{abstract}
Owing to its high protein secretion capacity, simple nutritional requirements, and GRAS (generally regarded as safe) status, Bacillus licheniformis is widely used as a host for the industrial production of enzymes, antibiotics, and peptides. However, as compared with its close relative Bacillus subtilis, little is known about the physiology and stress responses of B. licheniformis. To explore its temperature-stress metabolome, B. licheniformis strains ATCC 14580 and $\mathrm{B} 186$, with respective optimal growth temperatures of $42^{\circ} \mathrm{C}$ and $50^{\circ} \mathrm{C}$, were cultured at $42^{\circ} \mathrm{C}, 50^{\circ} \mathrm{C}$, and $60^{\circ} \mathrm{C}$ and their corresponding metabolic profiles were determined by gas chromatography/mass spectrometry and multivariate statistical analyses. It was found that with increased growth temperatures, the two B. licheniformis strains displayed elevated cellular levels of proline, glutamate, lysine, pentadecanoic acid, hexadecanoic acid, heptadecanoic acid, and octadecanoic acid, and decreased levels of glutamine and octadecenoic acid. Regulation of amino acid and fatty acid metabolism is likely to be associated with the evolution of protective biochemical mechanisms of $B$. licheniformis. Our results will help to optimize the industrial use of B. licheniformis and other important Bacillus species.
\end{abstract}

Keywords: Bacillus licheniformis, gas chromatography/mass spectrometry, temperature-stress metabolome, amino acid and fatty acid metabolism

\section{Introduction}

Bacillus licheniformis, a gram-positive endospore-forming bacterium, is commonly found in soil and other natural habitats. Owing to its high protein secretion capacity, simple nutritional requirements, and GRAS (generally regarded as safe) status, B. licheniformis is widely used as a host for the commercial production of important enzymes, antibiotics, peptides, etc. [1]. In such large-scale fermentation processes, $B$. licheniformis is exposed to a variety of stresses, such as low or high temperatures, oxidative stress, or osmotic challenges, which could hamper the quality and productivity of the fermentation product $[2,3]$. As compared with its close relative Bacillus subtilis, however, knowledge about the physiology and stress responses of B. licheniformis is still limited. Detailed knowledge of the stress response of B. licheniformis is therefore of paramount importance for industrial strain development.

To survive extreme environments, microorganisms have developed many strategies to react to such conditions. Among these strategies, acquisition of thermotolerance is mainly attributed to the activation and regulation of heat stress-related genes involved in the synthesis of specific compounds that protect the microorganisms from thermal stress. Previous studies of heat-resistant mechanisms of Saccharomyces cerevisiae, Escherichia coli, and B. subtilis have documented the synthesis and accumulation of specific compounds (e.g., glycerol, trehalose, misfolded proteins, amino acids and their derivatives) [4-6] that are activated by upregulated regulons, as well as structural and compositional 
changes in the cell wall and cytomembrane [4]. Since the publication of the genome sequences of $B$. licheniformis ATCC 14580 and DSM13 in 2004 [7, 8], several studies have been published with respect to revealing the resistance mechanisms of $B$. licheniformis against various stresses, such as oxidative [2], salt [9], heat, and ethanol stresses [6, 10]. Nevertheless, little information is available regarding the molecular mechanisms underlying its response to temperature stress.

As an essential component of the functional genomics approach, metabolomics is a recently developed powerful technology for the simultaneous identification and quantification of low-molecular-weight metabolites or intracellular metabolites, especially those involved in the cellular metabolism of an organism at a given time point under specific environmental conditions [11]. This technique has been widely applied to various research fields, including metabolic profiling [12], biological pathway characterization [13], and disease diagnosis [14]. Currently, there are several possible approaches that could be used for metabolic profiling. Among them, gas chromatography/mass spectrometry (GC/MS) has proved to be an efficient metabolomic tool and is widely used for the identification and quantification of metabolites based on its high peak resolution, reproducibility, and sensitivity [15]. Hence, the use of this approach for metabolic profiling may aid in understanding the response of $B$. licheniformis to temperature stress.

In the present study, B. licheniformis ATCC 14580 and B186 were cultivated at $42^{\circ} \mathrm{C}, 50^{\circ} \mathrm{C}$, and $60^{\circ} \mathrm{C}$, respectively. The previously established GC/MS-based metabolomic analysis [16] was then used to systematically determine the temporal changes in the metabolic profiles of B. licheniformis with multivariate statistical analyses as well as metabolic pathway analysis. Our results showed that there were significant differences in the profiles of amino acids and fatty acids for all the samples. This work identified a large amount of potential metabolic targets for further in-depth investigations of the acquired tolerance of B. licheniformis to temperature stress.

\section{Materials and Methods}

\section{Chemicals and Reagents}

Chromatogram-grade acetonitrile and methanol were supplied by Thermo Fisher Scientific Inc. (USA). Ribitol, pyridine, methoxyamine hydrochloride, and $N$-methyl- $N$-(trimethylsilyl) trifluoroacetamide (MSTFA) were purchased from Sigma-Aldrich Co. (USA). All other chemicals used in this study were commercially available in analytical grade.
Bacterial Strains and Determination of Their Optimum Growth Temperatures

B. licheniformis ATCC 14580 was supplied by the China Center for Type Culture Collection, and thermophilic strain B. lichenformis B186 that was screened according to the criteria described by Niu et al. [17] and stored at CICIM-CU (Jiangnan University, China) is usually used for the production of industrial enzymes [18]. An initial preculture $\left(12 \mathrm{~h}, 37^{\circ} \mathrm{C}\right.$, shaking at $\left.200 \mathrm{rpm}\right)$ of these two strains was performed in $250 \mathrm{ml}$ shake flasks containing $50 \mathrm{ml}$ of synthetic Belitzky minimal medium [19] with $0.2 \%(\mathrm{w} / \mathrm{v})$ glucose to reduce the differences caused by colony selection. Then, $1 \%(\mathrm{v} / \mathrm{v})$ of preculture was transferred to $50 \mathrm{ml}$ of fresh medium and cultivated at $37^{\circ} \mathrm{C}, 42^{\circ} \mathrm{C}, 50^{\circ} \mathrm{C}$, or $60^{\circ} \mathrm{C}$, respectively, with vigorous shaking until the stationary phase. Samples were withdrawn every $4 \mathrm{~h}$ to measure the optical cell density at $600 \mathrm{~nm}\left(\mathrm{OD}_{600}\right)$ to determine their optimum growth temperatures. All experiments were performed in triplicate from three independent cell cultures.

\section{Sample Preparation for GC/MS Analysis}

Cells of B. licheniformis ATCC 14580 and B186 were cultivated at $42^{\circ} \mathrm{C}, 50^{\circ} \mathrm{C}$, or $60^{\circ} \mathrm{C}$ until the stationary phase as described above. The fermentation broth of each sample was then withdrawn and adjusted to an $\mathrm{OD}_{600}$ of about 2.0. These 18 samples were classified into six groups and designated as A42, A50, A60, B42, B50, and B60, respectively. According to a previously established sample preparation method [16], $10 \mathrm{ml}$ of each cell suspension was thoroughly mixed with $30 \mathrm{ml}$ of pre-cooled $\left(<-40^{\circ} \mathrm{C}\right)$ quenching solution containing $60 \%(\mathrm{v} / \mathrm{v})$ methanol and $0.9 \%(\mathrm{v} / \mathrm{v})$ ammonium carbonate buffer. After centrifugation at $7,000 \times g$ and $4^{\circ} \mathrm{C}$ for $10 \mathrm{~min}$, the cell pellets collected were washed twice with $10 \mathrm{ml}$ of $0.9 \%(\mathrm{v} / \mathrm{v})$ ammonium carbonate buffer at $4^{\circ} \mathrm{C}$ and centrifuged at $5,000 \times g$ for $10 \mathrm{~min}$. All samples were snap frozen in liquid nitrogen and stored at $-80^{\circ} \mathrm{C}$ until further processing. All experiments were conducted in triplicate.

Intracellular metabolites of the 18 samples were extracted at $-20^{\circ} \mathrm{C}$ by the glass bead cell disruption method [16]. Briefly, all of the frozen samples were homogenized in $750 \mu$ l of ice-cold extraction solution, a mixture of methanol, acetonitrile, and distilled water $(2: 2: 1(\mathrm{v} / \mathrm{v} / \mathrm{v}))$. Then, the supernatant was collected by centrifugation at $12,000 \times g$ and $4^{\circ} \mathrm{C}$ for $10 \mathrm{~min}$. The remaining solid residues were further extracted using the same extract solution and intensively homogenized using a vortex. A second supernatant collected after centrifugation was pooled with the first one. The combined supernatants from the two extractions were concentrated to dryness by vacuum and kept at $-80^{\circ} \mathrm{C}$. A two-step methoximation/ silylation derivatization procedure [20] was then used to derivatize the samples for GC/MS. The dried samples were methoximated with $80 \mu \mathrm{l}$ of $40 \mathrm{mg} / \mathrm{ml}$ methoxyamine hydrochloride in anhydrous pyridine at $30^{\circ} \mathrm{C}$ for $90 \mathrm{~min}$. Subsequently, the samples were silylated with $80 \mu \mathrm{l}$ of MSTFA at $37^{\circ} \mathrm{C}$ for $120 \mathrm{~min}$, followed by the addition of $1 \mathrm{mg} / \mathrm{ml}$ of ribitol to the samples as derivatization standards.

Amino acids (lysine, phenylalanine, glutamate, methionine, and 
tyrosine), fatty acids (oleic acid, palmitic acid, lauric acid, and stearic acid), and a glucose reference standard were dissolved in pyridine to a final concentration of $200 \mu \mathrm{g} / \mathrm{ml}$. Then, $200 \mu \mathrm{l}$ of each reference standard was derivatized with the methodology described above and prepared for GC/MS analysis according to the procedure described below.

\section{GC/MS Analysis}

The derivatized extracts or derivatized reference standard were analyzed with an Agilent 7890A Gas Chromatography system coupled with an Agilent HP-5MS capillary column $(30 \times 0.25 \mathrm{~mm}$ ID, $0.25 \mu \mathrm{m}$ film thickness) with an Agilent 5975C-GC/MSD (Agilent Technologies, USA). A $1 \mu \mathrm{l}$ aliquot of the derivatives including derivatized standards was injected into the column in splitless mode. Helium was used as the carrier gas at a constant flow rate of $1.0 \mathrm{ml} / \mathrm{min}$, and the temperature of the inlet, transfer line, ion source, and quadrupole was controlled at $270^{\circ} \mathrm{C}, 280^{\circ} \mathrm{C}$, $230^{\circ} \mathrm{C}$, and $150^{\circ} \mathrm{C}$, respectively. The oven temperature was initially set to $70^{\circ} \mathrm{C}$, and $4 \mathrm{~min}$ after injection, it was increased to $200^{\circ} \mathrm{C}$ at a rate of $3^{\circ} \mathrm{C} / \mathrm{min}$, followed by a $10^{\circ} \mathrm{C} / \mathrm{min}$ ramp to $300^{\circ} \mathrm{C}$ and final 5 -min maintenance at $300^{\circ} \mathrm{C}$. The mass spectrometry was operated at electron impact ionization mode at $70 \mathrm{eV}$. Detection was achieved using an MS detector in an electron impact mode and a full-scan monitoring mode $(\mathrm{m} / \mathrm{z} 30-600)$, with an acquisition rate of 20 spectra/sec.

\section{Data Processing}

After analyzed by GC/MS, each sample or reference standard was represented by a typical GC/MS total ion current (TIC) chromatogram. MassHunter Workstation Data acquisition software (Agilent Technologies, USA) was used to operate the instrumentation. Chromatograms were deconvoluted into individual chemical peaks with the Molecular Feature Extraction algorithm in the MassHunter Qualitative Analysis software. Compounds were identified by comparison of the mass spectra with those available in the National Institute of Standards and Technology (NIST 11) nominal mass spectral library (http://www.nist.gov/srd/nistla.cfm) and customized reference mass spectral libraries [21]. The automated MS Deconvolution and Identification System was used for the identification and quantification of the metabolomics data. The mass spectra obtained were investigated carefully, and only those molecules with a matching probability of $>80 \%$ were considered. Within each sample, the retention time and $\mathrm{m} / \mathrm{z}$ data pairs were used to identify each peak, and the ion intensities for each peak detected were then normalized to the sum of the peak intensities in that sample. To account for any difference in concentration between samples, all data were normalized to a total value of 100 .

The normalized data were loaded to SIMCA-P (ver. 11.5) and transferred by autoscaling for further data processing. Statistical models including principal component analysis (unsupervised PCA and supervised orthogonal partial least squares discriminant analysis (OPLS-DA)) were constructed to identify marker metabolites in the data matrix [22]. PCA was first used to visualize the distribution of different samples for the evaluation of metabolic profiling. The discriminating metabolites were obtained from the OPLS-DA model where the metabolites with variable importance in the projection (VIP) values higher than 1 were selected. Statistical analysis was performed by $t$-test, and $p$ values of $<0.05$ were used to denote statistical significance.

\section{Metabolic Pathway Analysis}

To identify predicted metabolic pathways, the identified metabolites were uploaded to MBRole 2.0 (http:/ / csbg.cnb.csic.es/ mbrole2/), with Kyoto Encyclopedia of Genes and Genomes (KEGG) and Small Molecule Pathway Database (SMPDB) pathways as the annotations. The background set used in this study was B. licheniformis ATCC 14580. The metabolic pathway with a $p$ value of $<0.05$ was considered significantly affected by the differential metabolites.

\section{Results}

\section{Determining the Optimum Growth Temperatures of B. licheniformis ATCC 14580 and B186}

To determine the optimum growth temperatures of B. licheniformis ATCC 14580 and B186, both strains were cultivated in synthetic Belitzky minimal medium containing $0.2 \%(\mathrm{w} / \mathrm{v})$ glucose at $37^{\circ} \mathrm{C}, 42^{\circ} \mathrm{C}, 50^{\circ} \mathrm{C}$, or $60^{\circ} \mathrm{C}$, respectively, and the $\mathrm{OD}_{600}$ of the samples was measure at 4-h intervals. As shown in Fig. 1A, B. licheniformis ATCC 14580 grew optimally at $42^{\circ} \mathrm{C}$, and the greatest biomass $\left(\mathrm{OD}_{600}=19.07\right)$ was reached after 20-h cultivation. However, very slow growth of this stain was observed at $60^{\circ} \mathrm{C}$, with a maximum cell density of only 0.792 . The growth curve of B. licheniformis B186 cultivated at different temperatures is shown in Fig. 1B, and it showed optimum growth at $50^{\circ} \mathrm{C}$. This thermophilic strain also displayed better growth than B. lichniformis ATCC 14580 at $60^{\circ} \mathrm{C}$, and its $\mathrm{OD}_{600}$ reached a maximum of 3.86 within the first $12 \mathrm{~h}$. Besides this, B. licheniformis B186 exhibited higher growth rates than B. licheniformis ATCC 14580 at $50^{\circ} \mathrm{C}$ and $60^{\circ} \mathrm{C}$, whereas its growth rates at $37^{\circ} \mathrm{C}$ and $42^{\circ} \mathrm{C}$ were lower than those of B. licheniformis ATCC 14580.

\section{General Observations}

To investigate the temperature-stress metabolome of B. licheniformis, strains ATCC 14580 and B186 were cultivated at $42^{\circ} \mathrm{C}, 50^{\circ} \mathrm{C}$, or $60^{\circ} \mathrm{C}$, respectively, and the corresponding samples prepared for GS/MS analysis were classified into groups designated as A42, A50, A60, B42, B50, and B60, respectively. All of the 18 samples collected were analyzed by full-scan analysis using the GC/MS system. Representative 

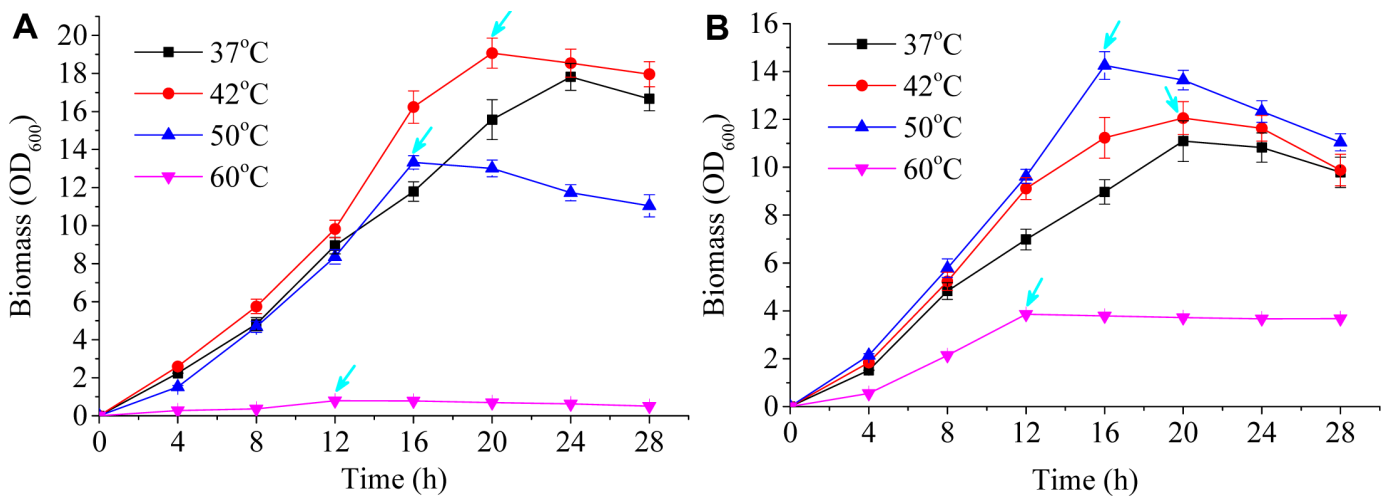

Fig. 1. Growth curves of B. licheniformis ATCC 14580 (A) and B186 (B) cultured at different temperatures.

As indicated by cyan arrows, the sampling time points for cultures at $42^{\circ} \mathrm{C}, 50^{\circ} \mathrm{C}$, and $60^{\circ} \mathrm{C}$ were 20,16 , and $12 \mathrm{~h}$, respectively.

GC/MS TIC chromatograms of the samples from B42, B50, and B60 are shown in Fig. S1. After data processing and acquisition, peak identification, and refining based on the $80 \%$ rule [23], a total of 110 metabolites out of 198 total peaks were identified (Table S1). Among these metabolites, amino acids, fatty acids, organic acids, carbohydrates, alkanes, and some small molecules (e.g., phosphate) were identified throughout the chromatographs.

\section{Determination of Potential Biomarkers in B. licheniformis B186}

Data from the B. licheniformis B186 samples were analyzed
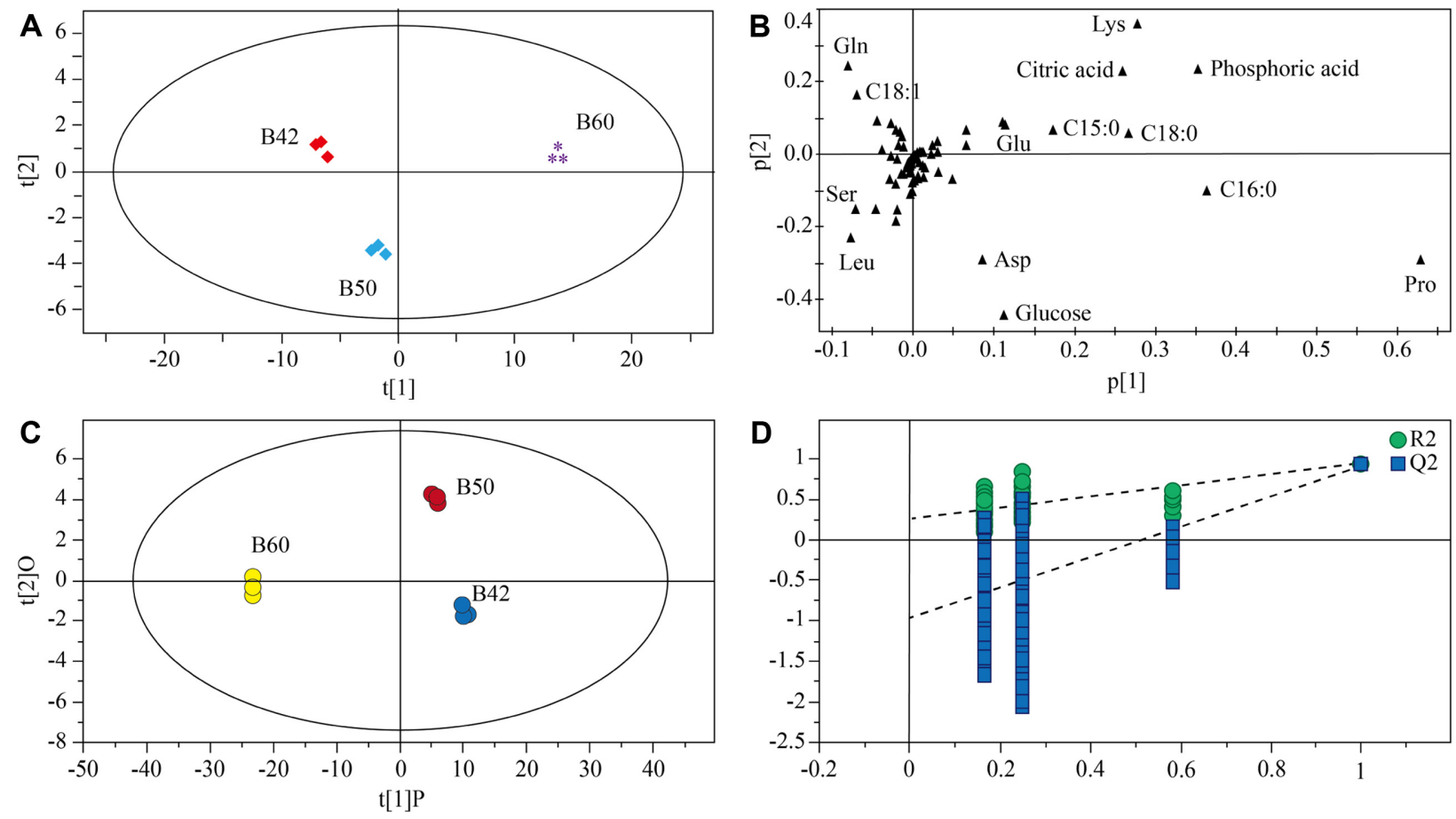

Fig. 2. Principal component analysis (unsupervised PCA and supervised orthogonal partial least squares discriminant analysis (OPLS-DA)) of marker metabolites in the data matrix.

Scores (A) and loadings plots (B) obtained by the PCA model. (C) Score plot obtained by the OPLS-DA model. Ser, Leu, Asp, Pro, Glu, Gln, Lys, C15:0, C16:0, C18:0, and C18:1 represent L-serine, L-leucine, L-aspartate, L-proline, L-glutamate, L-glutamine, L-lysine, pentadecanoic acid, hexadecanoic acid, octadecanoic acid, and octadecenoic acid, respectively. (D) The corresponding validation plot of the OPLS-DA model derived from the GC/MS metabolites of $\mathrm{B} 42$, B50, and B60, which represent samples of $B$. licheniformis $\mathrm{B} 186$ cultivated at $42^{\circ} \mathrm{C}, 50^{\circ} \mathrm{C}$, and $60^{\circ} \mathrm{C}$, respectively. 
by PCA and OPLS-DA models. Plotted PCA scores displayed relative obvious clustering for the three groups with the cumulative $\mathrm{R}^{2} \mathrm{X}$ of 0.928 and $\mathrm{Q}^{2}$ of 0.76 for this model (Fig. 2A). The first and second principal components ( $t 1$ and $t 2$ ) covered $57.7 \%$ and $35.1 \%$ of the total data variance, respectively, indicating the model had good ability of classification. PCA loading plot analysis revealed that the discrimination was mainly attributed to the metabolites such as L-lysine, L-glutamine, L-glutamate, citric acid, phosphonic acid, pentadecanoic acid, octadecanoic acid, octadecenoic acid, hexadecanoic acid, L-serine, L-leucine, L-aspartate, L-proline, and D-glucose (Fig. 2B). Similarly, the OPLS-DA scores plot exhibited distinct separation among the three groups with the cumulative $R^{2} X$ of 0.994 , $\mathrm{R}^{2} \mathrm{Y}$ of 0.979 , and $\mathrm{Q}^{2} \mathrm{Y}$ of 0.956 (Fig. 2C). The substantial separation of the samples from these three groups indicates the effect of temperature on the metabolic profile. Two hundred permutations of the cross-test validation of the OPLS-DA model showed $\mathrm{R}^{2}(0.0,0.27)$ and $\mathrm{Q}^{2}(0.0,-0.96)$, indicating satisfactory modeling and good discrimination (Fig. 2D).

On the basis of VIP values of metabolites derived from the OPLS-DA model and $t$-test discriminating among the B42, B50, and B60 groups, a total of 12 remarkable metabolites (VIP $>1$ and $p<0.05$ ) were selected as latent biomarkers, which were identified and are listed in Table 1. It was observed that the levels of a large number of amino acids and saturated fatty acids were increased in the B50 and $\mathrm{B} 60$ groups compared with that in the B42 group. In contrast, the levels of unsaturated fatty acids and a few amino acids were reduced as the cultivation temperature for B. licheniformis $\mathrm{B} 186$ increased from $42^{\circ} \mathrm{C}$ to $60^{\circ} \mathrm{C}$. In addition, as compared with group B42, the intensity of L-serine was increased in B50, but it was decreased in group B60.

\section{Identification of Latent Biomarkers in B. licheniformis ATCC 14580}

Data from the B. licheniformis ATCC 14580 samples were also analyzed using the previously described PCA and OPLS-DA models (data not shown). As shown in Table 2, according to the criteria for VIP statistics and $t$-test, a total of eight remarkable metabolites (VIP $>1$ and $p<0.05$ ) were identified and selected as potential biomarkers that contributed most toward discriminating among the A42, A50, and A60 groups. Interestingly, five of the metabolites detected were upregulated in A50 and A60 groups, whereas three were downregulated. These metabolites can be categorized into two classes: amino acids and fatty acids. Furthermore, the levels of L-proline were found to be increased to a greater extent in the $\mathrm{A} 60$ group, showing the greatest fold change (FC, 4.46), whereas L-glutamine was the metabolite whose levels showed the most severe decline (FC, 0.16).

\section{Discrepant Metabolite Comparison between B. licheniformis ATCC 14580 and B186}

To gain insight into stress-related metabolic variations

Table 1. Differential levels of metabolites revealed by the GC/MS chromatograph of B. licheniformis B186.

\begin{tabular}{|c|c|c|c|c|c|c|}
\hline Metabolites $^{\mathrm{a}}$ & Retention time (min) & Chemical class & VIP Value ${ }^{\mathrm{b}}$ & $p$ Value $^{c}$ & $\mathrm{FC}(\mathrm{B} 50 / \mathrm{B} 42)^{\mathrm{d}}$ & $\mathrm{FC}(\mathrm{B} 60 / \mathrm{B} 42)^{\mathrm{d}}$ \\
\hline L-Proline & 38.943 & Amino acid & 4.39 & 0.013 & 1.93 & 4.86 \\
\hline L-Glutamate & 51.373 & Amino acid & 3.75 & 0.005 & 2.87 & 5.20 \\
\hline L-Serine & 30.831 & Amino acid & 3.18 & 0.007 & 1.31 & 0.31 \\
\hline L-Lysine & 66.624 & Amino acid & 2.44 & 0.042 & 1.95 & 3.66 \\
\hline Octadecanoic acid & 70.325 & Fatty acid & 1.72 & 0.004 & 2.70 & 4.47 \\
\hline Hexadecanoic acid & 62.281 & Fatty acid & 1.70 & 0.002 & 1.79 & 3.10 \\
\hline D-Glucose & 58.964 & Carbohydrates & 1.61 & 0.000 & 1.17 & 1.69 \\
\hline L-Aspartate & 33.652 & Amino acid & 1.59 & 0.039 & 1.26 & 1.88 \\
\hline L-Glutamine & 44.132 & Amino acid & 1.58 & 0.006 & 0.75 & 0.24 \\
\hline Phosphonic acid & 51.911 & Inorganic acid & 1.50 & 0.033 & 2.67 & 4.58 \\
\hline Octadecenoic acid & 69.599 & Fatty acid & 1.17 & 0.028 & 0.84 & 0.35 \\
\hline
\end{tabular}

${ }^{a}$ Metabolites were identified using an available standard reference or NIST library database.

${ }^{\mathrm{b}}$ Variable importance in the projection (VIP) was obtained from OPLS-DA with a threshold of 1.0.

${ }^{\mathrm{c}}$ The $p$ value was calculated using the $t$-test (significance at $p<0.05$ ).

${ }^{\mathrm{d}} \mathrm{FC}$, fold change, the proportion of mean value of the peak area obtained from the B50 (or B60) group to that of the peak area obtained from the B42 group. 
Table 2. Differential levels of metabolites revealed by the GC/MS chromatograph of B. licheniformis ATCC 14580.

\begin{tabular}{lcccccc}
\hline \multicolumn{1}{c}{ Metabolites $^{\mathrm{a}}$} & Retention time $(\mathrm{min})$ & Chemical class & VIP value $^{\mathrm{b}}$ & $p$ Value $^{\mathrm{c}}$ & $\mathrm{FC}^{(\mathrm{A} 50 / \mathrm{A} 42)^{\mathrm{d}}}$ & $\mathrm{FC}_{(\mathrm{A} 60 / \mathrm{A} 42)^{\mathrm{d}}}$ \\
\hline L-Proline & 38.943 & Amino acid & 5.61 & 0.015 & 1.94 & 4.46 \\
L-Glutamine & 44.132 & Amino acid & 4.15 & 0.041 & 0.77 & 0.16 \\
L-Lysine & 66.624 & Amino acid & 2.06 & 0.032 & 1.27 & 1.53 \\
Hexadecanoic acid & 62.281 & Fatty acid & 2.04 & 0.044 & 1.21 & 1.17 \\
L-Glutamate & 51.373 & Amino acid & 1.21 & 0.007 & 1.12 & 1.96 \\
Octadecenoic acid & 69.599 & Fatty acid & 1.16 & 0.011 & 0.77 & 0.21 \\
Pentadecanoic acid & 51.749 & Fatty acid & 1.08 & 0.002 & 1.29 & 1.36 \\
Heptadecanoic acid & 66.153 & Fatty acid & 1.05 & 0.035 & 1.04 & 1.31 \\
\hline
\end{tabular}

${ }^{a}$ Metabolites were identified using an available standard reference or NIST library database.

${ }^{b}$ Variable importance in the projection (VIP) was obtained from OPLS-DA with a threshold of 1.0.

${ }^{\mathrm{c}}$ The $p$ value was calculated using the $t$-test (significance at $p<0.05$ ).

${ }^{\mathrm{d}} \mathrm{FC}$, fold change, mean value of the peak area obtained from the A50 (or A60) group/mean value of the peak area obtained from the A42 group.

between the type strain B. lichniformis ATCC 14580 and the thermophilic strain B. licheniformis B186, data of groups A42 and A60 samples were compared with those of B50 and B60, respectively. According to the VIP values of metabolites derived from the OPLS-DA model and $t$-test discriminating among the A42, B50, A60, and B60 groups (data not shown), a total of 10 candidate biomarkers (VIP $>1$ and $p<0.05)$ consisting of fatty acids and amino acids were obtained (Table 3). As compared with group A42 or A60 samples, the cellular levels of fatty acids and amino acids were found to be increased in the B50 and B60 groups, with the exception of glutamine. Additionally, the intensity of L-serine in group B50 was higher than that of L-serine in B42, but group B60 exhibited a lower intensity of L-serine than A60.

\section{Metabolic Pathway Analysis}

Combination of the results of Tables 1, 2, and 3 showed that nine metabolites are at least identified twice as latent biomarkers. Among them, the levels of proline, glutamate, lysine, pentadecanoic acid, hexadecanoic acid, heptadecanoic acid, and octadecanoic acid were shown to be increased with increased growth temperatures for B. licheniformis, whereas the levels of glutamine and octadecenoic acid underwent a significant decrease with increased growth temperatures. A functional pathway analysis facilitating further biological interpretation was subsequently performed by MBRole 2.0 to reveal the most relevant pathways in B. licheniformis. As shown in Table 4, the metabolic pathways significantly affected by these metabolites included arginine and proline metabolism, aminoacyl-tRNA biosynthesis,

Table 3. Differential levels of metabolites revealed by the GC/MS chromatographs of B. licheniformis ATCC 14580 and B186.

\begin{tabular}{|c|c|c|c|c|c|c|}
\hline Metabolites $^{a}$ & Retention time (min) & Chemical class & VIP value ${ }^{b}$ & $p$ Value $^{c}$ & $\mathrm{FC}(\mathrm{B} 50 / \mathrm{A} 42)^{\mathrm{d}}$ & $\mathrm{FC}(\mathrm{B} 60 / \mathrm{A} 60)^{\mathrm{d}}$ \\
\hline L-Proline & 38.943 & Amino acid & 6.82 & 0.043 & 1.53 & 4.52 \\
\hline Octadecanoic acid & 70.325 & Fatty acid & 4.21 & 0.035 & 1.61 & 2.47 \\
\hline Hexadecanoic acid & 62.281 & Fatty acid & 1.88 & 0.007 & 1.58 & 2.54 \\
\hline L-Glutamate & 51.373 & Amino acid & 1.65 & 0.004 & 1.08 & 4.04 \\
\hline Pentadecanoic acid & 51.749 & Fatty acid & 1.40 & 0.011 & 1.02 & 1.52 \\
\hline Heptadecanoic acid & 66.153 & Fatty acid & 1.29 & 0.022 & 1.14 & 1.83 \\
\hline L-Glutamine & 44.132 & Amino acid & 1.23 & 0.041 & 0.69 & 0.21 \\
\hline L-Leucine & 20.888 & Amino acid & 1.21 & 0.015 & 1.12 & 1.76 \\
\hline L-Serine & 30.831 & Amino acid & 1.17 & 0.002 & 1.21 & 0.83 \\
\hline L-Lysine & 66.624 & Amino acid & 1.04 & 0.010 & 1.54 & 3.02 \\
\hline
\end{tabular}

${ }^{a}$ Metabolites were identified using an available standard reference or NIST library database.

${ }^{b}$ Variable importance in the projection (VIP) was obtained from OPLS-DA with a threshold of 1.0.

${ }^{\mathrm{c}}$ The $p$ value was calculated using the $t$-test (significance at $p<0.05$ ).

${ }^{\mathrm{d}} \mathrm{FC}$, fold change, mean value of the peak area obtained from the B50 (or B60) group/mean value of the peak area obtained from the A42 (or A60) group. 
Table 4. Pathways significantly associated with the identified biomarkers.

\begin{tabular}{llcccc}
\hline \multicolumn{1}{c}{ Annotation } & \multicolumn{1}{c}{ Category $^{\mathrm{a}}$} & Set & In set & $p$ Value & FDR correction $^{\mathrm{b}}$ \\
\hline Arginine and proline metabolism & SMPDB pathways (YMDB) & 7 & 5 & $1.20 \mathrm{e}-07$ & $3.36 \mathrm{e}-06$ \\
Aminoacyl-tRNA biosynthesis & KEGG pathways & 6 & 4 & $3.27 \mathrm{e}-06$ & $7.52 \mathrm{e}-05$ \\
ABC transporters & KEGG pathways & 6 & 4 & $6.83 \mathrm{e}-06$ & $7.85 \mathrm{e}-05$ \\
Fatty acid biosynthesis & SMPDB pathways (YMDB) & 6 & 4 & $5.88 \mathrm{e}-05$ & $5.00 \mathrm{e}-04$ \\
\hline
\end{tabular}

${ }^{a}$ KEGG, Kyoto Encyclopedia of Genes and Genomes; SMPDB, Small Molecule Pathway Database; YMDB, Yeast Metabolite Database.

${ }^{b} \mathrm{FDR}$ correction is the adjusted $p$ value calculated as the false discovery rate (FDR).

amino acid metabolism, and fatty acid biosynthesis.

\section{Discussion}

The aim of the present study was to explore the temperature-stress metabolome of B. licheniformis using GC/MS-based metabolomic profiling. We examined the global metabolic changes of B. licheniformis ATCC 14580 and B186 cultured at different temperatures $\left(42^{\circ} \mathrm{C}, 50^{\circ} \mathrm{C}\right.$, and $60^{\circ} \mathrm{C}$ ). The results showed that cellular levels of some metabolites were significantly altered among groups B42, $\mathrm{B} 50$, and B60 of B. licheniformis B186, but the identified metabolites failed to show a similar changing pattern in B. licheniformis ATCC 14580 under the same conditions. Despite this inconsistency between the two strains examined, the affected metabolic pathways, particularly altered fatty acid and amino acid metabolism, could easily be identified as common responses to growth in increased temperatures for B. licheniformis (Fig. 3), which is also consistent with our previous findings [18].

The most significant alterations identified are changes in amino acid metabolism in B. licheniformis. As the growth temperature was increased from $42^{\circ} \mathrm{C}$ to $50^{\circ} \mathrm{C}$ and to $60^{\circ} \mathrm{C}$, decreased concentrations of L-glutamine were detected, whereas the levels of L-proline, L-glutamate, and L-lysine showed a significant increase. Bacteria usually accumulate high concentrations of compatible solutes (e.g., amines and amino acids) to achieve cellular protection under osmotic stress conditions $[12,25]$. In some microorganisms, such as B. subtilis [25] and E. coli [12], these compounds are also used as thermal stress protectants. Under conditions of depleted levels of naturally occurring osmolytes, a number of amino acids can help maintain the normal osmolarity of the cytoplasm and cellular water, increase the global thermostability of proteins, and prevent subcellular structures from collapse [12, 26].

Under thermal stress conditions, elevated levels of L-proline in B. licheniformis may be caused by the overexpression of gamma-glutamyl phosphate reductase, an enzyme involved

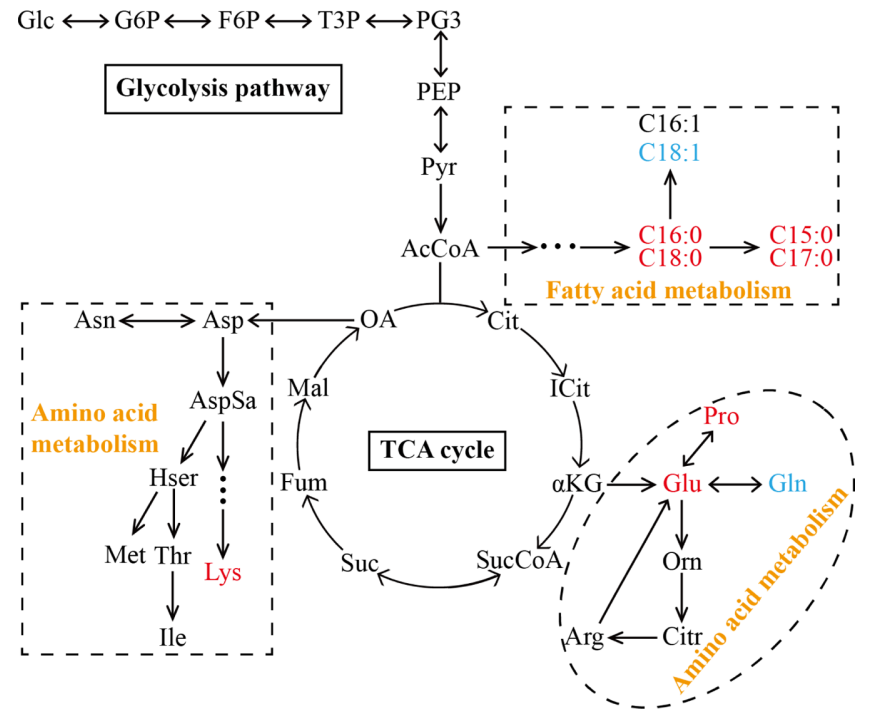

Fig. 3. Metabolic pathways related to the temperature stress of B. licheniformis.

The proposed pathway was obtained from the web-based KEGG pathway database (http://www.kegg.jp/) and the literature [24]. Metabolites colored in red, blue, or black represent higher, lower, or similar levels in heat-stressed B. licheniformis extracts compared with B. licheniformis cultivated at $42^{\circ} \mathrm{C}$. Glc, glucose; G6P, glucose 6-phosphate; F6P, fructose 6-phosphate; T3P, triose 3-phosphate; PG3, glycerate 3phosphate; PEP, phosphoenolpyruvate; $\mathrm{Pyr}$, pyruvate; $\mathrm{AcCoA}$, acetyl coenzyme A; Cit, citrate; ICit, isocitrate; $\alpha \mathrm{KG}$, $\alpha$-ketoglutarate; SucCoA, succinate coenzyme A; Suc, succinate; Fum, fumarate; Mal, malate; OA, oxaloacetate; Pro, L-proline; Glu, L-glutamate; Gln, L-glutamine; Orn, ornithine; Citr, citruline; Arg, L-arginine; Asp, L-asparate; Asn, L-asparagine; AspSa, aspartate semialdehyde; Hser, homo-L-serine; Met, L-methionine; Thr, L-threonine; Ile, L-isoleucine; C15:0, pentadecanoic acid; C16:0, hexadecanoic acid; C16:1, hexadecenoic acid; C17:0, heptadecanoic acid; C18:0, octadecanoic acid; C18:1, octadecenoic acid.

in the biosynthesis of L-proline from L-glutamate [18]. High concentrations of L-proline were usually produced in response to osmotic stress [3, 9, 27], and the enzymes involved in this process have been extensively studied in B. subtilis $[25,28]$. Aside from acting as an osmostress protectant, L-proline can also be exploited by B. licheniformis 
as a sole source of carbon and nitrogen [9]. L-Glutamate is not only the precursor for L-proline synthesis in B. licheniformis (Fig. 2) and other bacteria, but also exhibits an associated thermoprotective effect to enable protein disaggregation and refolding under high temperatures in E. coli cells [29]. In addition, L-glutamine is a primary precursor for the biosynthesis of stress-related metabolites, such as polyamines and $\gamma$-aminobutyrate [12]. In response to stress, L-lysine could be converted to L-glutamate and other stress-related metabolites, such as putrescine and $\gamma$-aminobutyrate, and thus its catabolism was highly affected by stress [30]. The depleted level of L-glutamine in B. licheniformis under high growth temperatures may be caused by the accumulation of L-proline.

Altered fatty acid metabolism in B. licheniformis was another key finding in this study. The levels of a wide spectrum of fatty acids, especially saturated fatty acids such as pentadecanoic acid, hexadecanoic acid, heptadecanoic acid, and octadecanoic acid, were found to be significantly increased with increased cultivation temperatures. In contrast, the levels of unsaturated fatty acids (e.g., octadecenoic acid) showed a significant decrease.

In diverse organisms ranging from bacteria to humans, the ratio between disordered (fluid) lipids and ordered (non-fluid) lipids within a lipid bilayer is referred to as the fluidity of the membrane. Temperature stress affects the fluidity of cytoplasmic membranes in living cells. Heat causes fluidization of membranes, which can be compensated by the replacement of the unsaturated fatty acids in membrane lipids with the de novo synthesized saturated fatty acids and by the synthesis of the membrane-stabilizing proteins. Alternatively, cold causes a decrease in the fluidity of the membrane (membrane rigidification). This is compensated by increasing the degree of fatty acid unsaturation and monounsaturated straight-chains [31, 32]. This thermal regulation system can adapt the membrane lipids for optimal behavior at new growth temperatures. Moreover, this kind of adjustment of membrane fatty acid composition has already been reported in a large number of B. subtilis species [31, 33, 34]. However, these findings are inconsistent with the upregulation of acyl carrier protein under heat stress conditions, which participates in the biosynthesis of unsaturated fatty acids [18]. Further investigations are therefore needed to fully characterize the molecular mechanisms underlying temperature-induced changes in membrane fluidity.

In summary, the combined use of the GC/MS analytical method and multivariate statistics facilitates the identification and discrimination of metabolic shifts with statistical significance in samples of B. licheniformis ATCC 14580 and B186 cultivated at different temperatures. Dramatic changes in amino acid and fatty acid metabolism were observed. Biological validation through further experimentation is required to gain better insights into the specific temperaturestress physiology of B. licheniformis. A better understanding of the molecular mechanisms underlying the response of B. licheniformis to temperature stress would inform the design of robust industrial strains with improved growth temperature profiles for $B$. licheniformis and other important Bacillus species.

\section{Acknowledgments}

This work was financially supported by the National Natural Science Foundation of China (Grant No. 31370076), the International Collaborative Project Supported by National Natural Science Foundation of China (NSFC) and National Research Foundation of South Africa (NFC) (Grant No. 31461143026), and the Youth Innovation Fund from Tianjin University of Science \& Technology (Grant No. 2016LG15).

\section{Conflict of Interest}

The authors have no financial conflicts of interest to declare.

\section{References}

1. Schallmey M, Singh A, Ward OP. 2004. Developments in the use of Bacillus species for industrial production. Can. J. Microbiol. 50: 1-17.

2. Schroeter R, Voigt B, Jürgen B, Methling K, Pöther DC, Schäfer $\mathrm{H}$, et al. 2011. The peroxide stress response of Bacillus licheniformis. Proteomics 11: 2851-2866.

3. Voigt B, Schroeter R, Schweder T, Jurgen B, Albrecht D, van Dijl JM, et al. 2014. A proteomic view of cell physiology of the industrial workhorse Bacillus licheniformis. J. Biotechnol. 191: 139-149.

4. Blaby IK, Lyons BJ, Wroclawska-Hughes E, Phillips GC, Pyle TP, Chamberlin SG, et al. 2012. Experimental evolution of a facultative thermophile from a mesophilic ancestor. Appl. Environ. Microbiol. 78: 144-155.

5. Sola-Penna M, Meyer-Fernandes JR. 1998. Stabilization against thermal inactivation promoted by sugars on enzyme structure and function: why is trehalose more effective than other sugars? Arch. Biochem. Biophys. 360: 10-14.

6. Voigt B, Schroeter R, Jürgen B, Albrecht D, Evers S, Bongaerts J, et al. 2013. The response of Bacillus licheniformis to heat and ethanol stress and the role of the SigB regulon. 
Proteomics 13: 2140-2161.

7. Rey MW, Ramaiya P, Nelson BA, Brody-Karpin SD, Zaretsky EJ, Tang M, et al. 2004. Complete genome sequence of the industrial bacterium Bacillus licheniformis and comparisons with closely related Bacillus species. Genome Biol. 5: r77.

8. Veith B, Herzberg C, Steckel S, Feesche J, Maurer KH, Ehrenreich $\mathrm{P}$, et al. 2004. The complete genome sequence of Bacillus licheniformis DSM13, an organism with great industrial potential. J. Mol. Microbiol. Biotechnol. 7: 204-211.

9. Schroeter R, Hoffmann T, Voigt B, Meyer H, Bleisteiner M, Muntel J, et al. 2013. Stress responses of the industrial workhorse Bacillus licheniformis to osmotic challenges. PLoS One 8: e80956.

10. Nielsen AK, Breüner A, Krzystanek M, Andersen JT, Poulsen TA, Olsen PB, et al. 2010. Global transcriptional analysis of Bacillus licheniformis reveals an overlap between heat shock and iron limitation stimulon. J. Mol. Microbiol. Biotechnol. 18: 162-173.

11. Song $\mathrm{H}$, Wang $\mathrm{L}$, Liu HL, Wu XB, Wang HS, Liu ZH, et al. 2011. Tissue metabolomic fingerprinting reveals metabolic disorders associated with human gastric cancer morbidity. Oncol. Rep. 26: 431-438.

12. Ye Y, Zhang L, Hao F, Zhang J, Wang Y, Tang H. 2012. Global metabolomic responses of Escherichia coli to heat stress. J. Proteome Res. 11: 2559-2566.

13. Nicholson JK, Connelly J, Lindon JC, Holmes E. 2002. Metabonomics: a platform for studying drug toxicity and gene function. Nat. Rev. Drug Discov. 1: 153-161.

14. Denery JR, Nunes AA, Hixon MS, Dickerson TJ, Janda KD. 2010. Metabolomics-based discovery of diagnostic biomarkers for onchocerciasis. PLoS Negl. Trop. Dis. 4: e834.

15. Putri SP, Yamamoto S, Tsugawa H, Fukusaki E. 2013. Current metabolomics: technological advances. J. Biosci. Bioeng. 116: 9-16.

16. Wang H, Chen Z, Yang J, Liu Y, Lu F. 2015. Optimization of sample preparation for the metabolomics of Bacillus licheniformis by GCMS, pp. 579-588. In Zhang T-C, Nakajima M (eds.). Advances in Applied Biotechnology. Proceedings of the 2nd International Conference on Applied Biotechnology (ICAB 2014), Vol. I. Springer, Berlin-Heidelberg.

17. Niu D, Zuo Z, Shi GY, Wang ZX. 2009. High yield recombinant thermostable $\alpha$-amylase production using an improved Bacillus licheniformis system. Microb. Cell Fact. 8: 611-631.

18. Dong Z, Chen Z, Wang H, Tian K, Jin P, Liu X, et al. 2017. Tandem mass tag-based quantitative proteomics analyses reveal the response of Bacillus licheniformis to high growth temperatures. Ann. Microbiol. 67: 501-510.

19. Stülke J, Hanschke R, Hecker M. 1993. Temporal activation of $\beta$-glucanase synthesis in Bacillus subtilis is mediated by the GTP pool. Microbiology 139: 2041-2045.

20. Halket JM, Waterman D, Przyborowska AM, Patel RK, Fraser PD, Bramley PM. 2005. Chemical derivatization and mass spectral libraries in metabolic profiling by GC/MS and LC/MS/MS. J. Exp. Bot. 56: 219-243.

21. Denkert C, Budczies J, Kind T, Weichert W, Tablack P, Sehouli J, et al. 2006. Mass spectrometry-based metabolic profiling reveals different metabolite patterns in invasive ovarian carcinomas and ovarian borderline tumors. Cancer Res. 66: 10795-10804.

22. Bundy JG, Willey TL, Castell RS, Ellar DJ, Brindle KM. 2005. Discrimination of pathogenic clinical isolates and laboratory strains of Bacillus cereus by NMR-based metabolomic profiling. FEMS Microbiol. Lett. 242: 127-136.

23. Bijlsma S, Bobeldijk I, Verheij ER, Ramaker R, Kochhar S, Macdonald IA, et al. 2006. Large-scale human metabolomics studies: a strategy for data (pre-) processing and validation. Anal. Chem. 78: 567-574.

24. Çalık P, Çalık G, Özdamar TH. 2001. Bioprocess development for serine alkaline protease production: a review. Rev. Chem. Eng. 17: 1-62.

25. Hoffmann T, Bremer E. 2011. Protection of Bacillus subtilis against cold stress via compatible-solute acquisition. J. Bacteriol. 193: 1552-1562.

26. Neelon K, Schreier HJ, Meekins H, Robinson PM, Roberts MF. 2005. Compatible solute effects on thermostability of glutamine synthetase and aspartate transcarbamoylase from Methanococcus jannaschii. Biochim. Biophys. Acta 1753: 164-173.

27. Bursy J, Pierik AJ, Pica N, Bremer E. 2007. Osmotically induced synthesis of the compatible solute hydroxyectoine is mediated by an evolutionarily conserved ectoine hydroxylase. J. Biol. Chem. 282: 31147-31155.

28. Zaprasis A, Hoffmann T, Wunsche G, Florez LA, Stulke J, Bremer E. 2014. Mutational activation of the RocR activator and of a cryptic rocDEF promoter bypass loss of the initial steps of proline biosynthesis in Bacillus subtilis. Environ. Microbiol. 16: 701-717.

29. Diamant S, Eliahu N, Rosenthal D, Goloubinoff P. 2001. Chemical chaperones regulate molecular chaperones in vitro and in cells under combined salt and heat stresses. J. Biol. Chem. 276: 39586-39591.

30. Galili G, Tang G, Zhu X, Gakiere B. 2001. Lysine catabolism: a stress and development super-regulated metabolic pathway. Curr. Opin. Plant Biol. 4: 261-266.

31. Suutari M, Laakso S. 1994. Microbial fatty acids and thermal adaptation. Crit. Rev. Microbiol. 20: 285-328.

32. Los DA, Mironov KS, Allakhverdiev SI. 2013. Regulatory role of membrane fluidity in gene expression and physiological functions. Photosynth. Res. 116: 489-509.

33. Mansilla MC, de Mendoza D. 2005. The Bacillus subtilis desaturase: a model to understand phospholipid modification and temperature sensing. Arch. Microbiol. 183: 229-235.

34. Sikorski J, Brambilla E, Kroppenstedt RM, Tindall BJ. 2008. The temperature-adaptive fatty acid content in Bacillus simplex strains from 'Evolution Canyon', Israel. Microbiology 154: $2416-2426$ 